\title{
Operative Entfernung von Weisheitszähnen
}

\author{
M. Kunkel $\cdot$ J. Becker $\cdot$ P. Boehme $\cdot$ P. Engel $\cdot$ G. Göz $\cdot$ D. Haessler $(\dagger) \cdot$ D. Heidemann $\cdot$ E. Hellwig $\cdot$ I. Kopp $\cdot$ \\ B. Kreusser $\cdot$ H.-Ch. Lauer $\cdot$ H. Luckey $\cdot$ E. Reinhard $\cdot$ P. Schopf $\cdot$ R. Singer $\cdot$ H. Terheyden $\cdot$ J. C. Türp $\cdot$ \\ M. Weber $\cdot$ D. Weingart $\cdot$ R. Werkmeister $\cdot$ W. Wagner
}

Online veröffentlicht: 14 Dezember 2006

(C) Springer-Verlag 2006

\section{Mund Kiefer GesichtsChir (2006) 10: 205-211}

Der Autor des Artikels Martin Kunkel hat dem SpringerVerlag irrtümlich die Rechte übertragen. Inhaber der Rechte sind aufgrund der tatsächlichen Sachlage auch die Zahnärztliche Zentralstelle Qualitätssicherung und die DGMKG. Die Copyrightzeile hätte lauten müssen:

(C) Zahnärztliche Zentralstelle Qualitätssicherung, DGMKG und Springer-Verlag 2006

Die Adresse "Mainz" gilt nur für den korrespondierenden Autor (Kunkel) und für Herrn Prof. Wagner. Alle anderen Autoren werden hier noch einmal genannt:

Becker, J. (Leitliniengruppe: Dentoalveoläre Chirurgie)

Boehme, P. (Ausschuss Qualität in der Zahnmedizin der Bundeszahnärztekammer)

Engel, P. (Bundeszahnärztekammer)

Göz, G. (Deutsche Gesellschaft für Kieferorthopädie)

Haessler, D. (Leitliniengruppe: Dentoalveoläre Chirurgie)

Heidemann, D. (Deutsche Gesellschaft für Zahn-, Mundund Kieferheilkunde)

Die Online-Version des Originalartikels befindet sich unter: http://dx.doi.org/10.1007/s10006-006-0007-7

M. Kunkel ( $)$ J. Becker · P. Boehme · P. Engel · G. Göz ·

D. Haessler ( $\dagger$ ) D D. Heidemann · E. Hellwig · I. Kopp · B. Kreusser ·

H.-Ch. Lauer · H. Luckey · E. Reinhard · P. Schopf - R. Singer ·

H. Terheyden · J. C. Türp · M. Weber · D. Weingart · R. Werkmeister ·

W. Wagner

Johannes Gutenberg-Universität Mainz, Klinik für Mund-, Kiefer-

und Gesichtschirurgie,

Augustusplatz 2, 55131 Mainz, Germany

E-Mail: kunkel@mkg.klinik.uni-mainz.de

Tel.: +49-6131-175458

Fax: +49-6131-176602
Hellwig, D. (Deutsche Gesellschaft für Zahnerhaltung) Kopp, I. (AWMF)

Kreusser, B. (Leitliniengruppe: Dentoalveoläre Chirurgie) Lauer, H.-Ch. (Deutsche Gesellschaft für Zahnärztliche Prothetik und Werkstoffkunde)

Luckey, H. (Berufsverband Deutscher Oralchirurgen)

Reinhard, E. (Leitliniengruppe: Dentoalveoläre Chirurgie)

Schopf, P. (Deutsche Gesellschaft für Kieferorthopädie)

Singer, R. (Leitliniengruppe: Dentoalveoläre Chirurgie)

Terheyden, H. (Leitliniengruppe: Dentoalveoläre Chirurgie)

Türp, J.C. (Deutsche Gesellschaft für Zahnärztliche Prothetik und Werkstoffkunde)

Weber, M. (Kassenzahnärztliche Bundesvereinigung)

Weingart, D. (Leitliniengruppe: Dentoalveoläre Chirurgie)

Werkmeister, R. (Leitliniengruppe: Dentoalveoläre Chirurgie) 